\title{
TOTAL STABILITY IN NONDIFFERENTIABLE SYSTEMS
}

\author{
ROGER C. MCCANN
}

\begin{abstract}
A definition of total stability in nondifferentiable dynamical systems is given. A prolongation is defined which characterizes the total stability of compact sets. A compact set which is the intersection of compact asymptotically stable sets is shown to be totally stable.
\end{abstract}

Let $M$ be a compact invariant (with respect to a dynamical system $\pi$ ) subset of a locally compact metric space $X$. Stability properties of $M$ may be described in terms of a fundamental system of neighborhoods of $M$, e.g. $M$ is stable if and only if $M$ possesses a fundamental system of positively invariant neighborhoods; $M$ is absolutely stable if and only if $M$ possesses a fundamental system of absolutely stable neighborhoods [1]. In [4] it is proved that $M$ is absolutely stable if and only if $M$ possesses a fundamental system $\mathscr{F}$ of neighborhoods such that

(i) If $U \in \mathscr{F}$, then $U$ is open and positively invariant,

(ii) if $U, V \in \mathscr{F}$ are such that $\bar{U} \subset V$, then there is a $W \in \mathscr{F}$ such that $\bar{U} \subset W \subset \bar{W} \subset V$.

A similar theorem can be proved where $\mathscr{F}$ consists of stable neighborhoods of $M$. Thus the absolute stability of $M$ can be described in terms of fundamental systems of neighborhoods which consist of positively invariant, stable, or absolutely stable neighborhoods of $M$. Since four of the basic concepts of dynamical system theory are invariance, stability, absolute stability, and asymptotic stability, a natural question which arises is: "What type of stability does $M$ have if it possess a fundamental system of asymptotically stable neighborhoods?" Obviously $M$ will be absolutely stable, but are there any other stability characteristics which are not typical of a compact absolutely stable set? It is the purpose of this paper to identify this type of stability, which we will call total stability.

In [5] and [6] Seibert defines a type of stability ("rough" stability or $P^{*}$ stability) which requires that the set under consideration possess a fundamental system of asymptotically stable neighborhoods. We do not use this definition, but do show that a compact set which is $P^{*}$ stable is also totally stable.

Received by the editors April 12, 1971 and, in revised form, January 7, 1972.

AMS 1970 subject classifications. Primary 34C35, 34D10; Secondary 34D30.

Key words and phrases. Dynamical system, total stability, prolongation, asymptotic stability.

c) American Mathematical Society 1972 
The idea of "total stability", "stability under persistent perturbations" or "strict stability" has been studied by many authors. (For a bibliography see [1], [5], [6].) The basic concept may be summarized as follows: A class of dynamical systems is given, one of which, $\pi_{\mathbf{0}}$, is considered as being "unperturbed" while the others are considered perturbed relative to $\pi_{0}$. A set $M$ is called "totally stable" if all trajectories of dynamical systems sufficiently close to $\pi_{0}$, with initial points sufficiently near $M$, remain within a given neighborhood of $M$.

In what follows $R$ and $R^{+}$will denote the reals and nonnegative reals respectively.

A dynamical system on a topological space $X$ is a mapping $\pi$ of $X \times R$ into $X$ such that (where $x \pi t=\pi(x, t)$ for $(x, t) \in X \times R$ )

(i) $x \pi 0=x$ for every $x \in X$,

(ii) $(x \pi t) \pi s=x \pi(t+s)$ for every $x \in X$ and $s, t \in R$,

(iii) $\pi$ is continuous in the product topology.

Let $A \subset X$ and $B \subset R$. Then $A \pi B$ will denote the set $\{x \pi t: x \in A, t \in B\}$. A subset $A$ of $X$ is called positively invariant if and only if $A \pi R^{+}=A$.

A subset $A$ of $X$ is called stable if for any neighborhood $U$ of $A$ there is a neighborhood $V$ of $A$ such that $V \pi R^{+} \subset U$. The set $A$ is called asymptotically stable if it is stable and there is a neighborhood $W$ of $A$ such that for every $x \in W$, the positive limit set of $x$ is a subset of $A$, i.e. $\bigcap\{\operatorname{cl}(x \pi[t,+\infty)): t \geqq 0\} \subset A$ for every $x \in W$.

Let $M$ be a compact subset of $X$. A Liapunov function $f$ for $M$ is a continuous mapping of a neighborhood $W$ of $M$ into $R^{+}$such that

(i) $f(x)=0$ if and only if $x \in M$,

(ii) $f(x \pi t) \leqq f(x)$ for $x \notin M, t>0$, and $x \pi[0, t] \subset W$.

A compact set $M$ is asymptotically stable if and only if there exists a Liapunov function $f$ for $M$ such that $f(x \pi t)<f(x)$ wherever $x \notin M$ and $t>0[1]$.

A subset $S$ of $X$ is called a section with respect to $\pi$ if $(S \pi t) \cap S=\varnothing$ for all $t \neq 0$.

In this paper we will be concerned with a dynamical system $\pi$ and net $\pi_{i}$ of dynamical systems on $X$ such that $\pi_{i} \rightarrow \pi$ in the sense that if $x_{i} \rightarrow x$ and $t_{i} \rightarrow t$, then $x_{i} \pi_{i} t_{i} \rightarrow x \pi t$. If $\pi_{i} \rightarrow \pi$, we say that $\pi_{i}$ converges to $\pi$. If $X$ is locally compact, then the convergence of $\pi_{i}$ to $\pi$ as defined above is equivalent to the convergence of $\pi_{i}$ to $\pi$ in the compact open topology [2, VI, 3.3].

Definition 1. A subset $M$ of $X$ is totally stable (with respect to $\pi$ ) if and only if given any neighborhood $U$ of $M$ there is another neighborhood $V$ of $M$ such that, for any net $\pi_{i}$ of dynamical systems on $X$ which converges to $\pi, V \pi_{i} R^{+}$is eventually a subset of $U$.

It should be noted that total stability and absolute stability are distinct 
concepts. Let $\pi_{\varepsilon}, \varepsilon>0$, be the planar dynamical system given by $\dot{x}=$ $\left[\begin{array}{cc}\varepsilon & 1 \\ -1 & 0\end{array}\right] x$. For $\varepsilon=0$, the origin is a center and, hence, absolutely stable. For $\varepsilon>0$, the origin is globally negatively asymptotically stable. Clearly $\pi_{\varepsilon} \rightarrow \pi_{0}$ as $\varepsilon \rightarrow 0$. The origin is absolutely stable, but not totally stable.

Let $\Pi$ denote the set of all dynamical systems on $X$ and $\pi \in \Pi$. $\pi$ is the dynamical system which will be considered as unperturbed throughout this paper.

Definition 2. A prolongation [1] with respect to $\pi \in \Pi$ is a mapping $Q$ of $x$ into $2^{x}$ satisfying

(a) if $x \in X$, then $x \pi R^{+} \subset Q(x)$,

(b) $Q(x)=\bigcap\{\operatorname{cl}(Q(W)): W$ a neighborhood of $x\}$,

(c) if $A$ is a compact set and $x \in A$, then $Q(x) \subset A$ or $Q(x) \cap \partial A \neq \varnothing$.

If, in addition, $Q^{2}=Q$, then $Q$ is called a transitive prolongation.

Evidently (b) is equivalent to

(b) $Q(x)=\left\{y\right.$ :there are nets $x_{i} \rightarrow x, y_{i} \rightarrow y$, with $\left.y_{i} \in Q\left(x_{i}\right)\right\}$.

Define $P: X \rightarrow 2^{x}$ as follows

$P(x)=\left\{y:\right.$ there exist nets $x_{i}$ in $X, \pi_{i}$ in $\Pi$, and $t_{i}$ in $R^{+}$

such that $x_{i} \rightarrow x, \pi_{i} \rightarrow \pi$, and $\left.x_{i} \pi_{i} t_{i} \rightarrow y\right\}$.

Evidently $P$ satisfies (a).

LeMma 3. P satisfies (b).

Proof. Obviously, $P(x) \subset \bigcap\{\operatorname{cl}(P(W)): W$ a neighborhood of $x\}$. We will prove the opposite inclusion. Let $y \in \bigcap\{\operatorname{cl}(P(W)): W$ a neighborhood of $x\}$. Then there are nets $x_{i}$ and $y_{i}$ in $X$ such that $x_{i} \rightarrow x, y_{i} \rightarrow y$, and $y_{i} \in$ $P\left(x_{i}\right)$. Thus there are nets $x_{i}^{j} \rightarrow^{j} x_{i}, \pi^{j} \rightarrow \pi$, and $t^{j}$ such that $x_{i}^{j} \pi^{j} t^{j} \rightarrow^{j} y_{i}$. Then [3, p. 69], there are nets $x_{k} \rightarrow x, \pi_{k} \rightarrow \pi$, and $t_{k}$ such that $x_{k} \pi_{k} t_{k} \rightarrow y$. Thus $y \in P(x)$, which implies the desired result.

LEMMA 4. P satisfies (c).

Proof. Let $A$ be compact and $x \in A$. If $x \in \partial A$, then $x \in x \pi R^{+} \subset P(x)$, so that $P(x) \cap \partial A \neq \varnothing$. Now suppose that $x \in$ int $A$ and that $P(x) \notin A$. Then there is a $y \in P(x)-A$ and nets $x_{i}, \pi_{i}$ and $t_{i}$ such that $x_{i} \rightarrow x, \pi_{i} \rightarrow \pi$, and $x_{i} \pi_{i} t_{i} \rightarrow y$. Eventually $x_{i} \in$ int $A$ and $x_{i} \pi_{i} t_{i} \in X-A$. Since $\pi_{i}$ is continuous there is a $\tau_{i}, 0<\tau_{i}<t_{i}$, such that eventually $x_{i} \pi_{i} \tau_{i} \in \partial A$. The compactness of $\partial A$ implies that a subnet of $x_{i} \pi_{i} t_{i}$ converges to a $z \in \partial A$. Then $z \in P(x) \cap \partial A$. This completes the proof.

Combining the previous lemmas we have

THEOREM 5. $P$ is a prolongation.

THEOREM 6. A compact set $M$ in a locally compact space is totally stable if and only if $P(M)=M$. 
Proof. Suppose that $P(M)=M$ and that $M$ is not totally stable. Then there is a compact neighborhood $U$ of $M$ such that for each neighborhood $V$ of $M$, there is a net $\pi_{v}^{i} \rightarrow \pi$ such that $V \pi_{v}^{i} R^{+}$is not eventually a subset of $U$. Hence there are nets $x_{i} \rightarrow x \in M, \pi_{i} \rightarrow \pi$, and $t_{i}$ such that $x_{i} \pi_{i} t_{i} \in X-U$. Then there is a net $\tau_{i}$ such that $x_{i} \pi_{i} \tau_{i} \in \partial U$. The compactness of $\partial U$ implies $P(x) \cap \partial U \neq \varnothing$. This contradiction implies that if $P(M)=M$, then $M$ is totally stable.

Now suppose that $M$ is totally stable and let $U$ be any neighborhood of $M$. Then there exists a neighborhood $V \subset U$ of $M$ such that for any net $\pi_{i}$ in $\Pi$, with $\pi_{i} \rightarrow \pi$, eventually $V \pi_{i} R^{+} \subset U$. It follows that $P(M) \subset U$, which implies $P(M)=M$ since $U$ was an arbitrary neighborhood of $M$. This completes the proof.

COROLlary 7. Let $M_{i}$ be a family of compact totally stable sets in a locally compact space. Then $M=\bigcap M_{i}$ is totally stable.

Proof. $M \subset P(M)=P\left(\bigcap M_{i}\right) \subset \bigcap P\left(M_{i}\right)=\bigcap M_{i}=M$.

THEOREM 8. In a locally compact space a compact set $M$ is totally stable if and only if for every neighborhood $U$ of $M$, there is another neighborhood $V$ of $M$ such that $P(V) \subset U$.

Proof. Suppose $M$ is totally stable, i.e. $P(M)=M$, and that there is a neighborhood $U$ of $M$ such that for every neighborhood $V$ of $M, P(V) \notin U$. Without loss of generality we may assume that $U$ is compact. Then there are nets $x_{i} \rightarrow x \in M$ and $y_{i}$ such that $y_{i} \in P\left(x_{i}\right)$ and $y_{i} \notin U$. By property (c) (Definition 2) there exist $z_{i} \in \partial U \cap P\left(x_{i}\right)$. Since $\partial U$ is compact, a subnet of $z_{i}$ converges to a point $z \in \partial U$. Then $z \in P(x) \cap \partial U$. This contradiction implies that there is a neighborhood $V$ of $M$ such that $P(V) \subset U$.

To prove the converse let $U$ and $V$ be neighborhoods of $M$ such that $P(V) \subset U$. Then $M \subset P(M) \subset P(V) \subset U$. Since $U$ was an arbitrary neighborhood of $M$ we have $P(M)=M$. This completes the proof.

THEOREM 9. In a locally compact space a compact asymptotically set $M$ is totally stable.

Proof. Let $f$ be a Liapunov function for $M$. Then $\left\{f^{-1}([0, r]): r \in R^{+}\right\}$ is a fundamental system of neighborhoods of $M$. Moreover, since $\partial\left(f^{-1}([0, r])\right)=f^{-1}(r)$ and $f$ is strictly decreasing along trajectories, we have that $\partial\left(f^{-1}([0, r])\right)$ is a section. Now let $U$ be any compact neighborhood and $V$ be any member of the above fundamental system of neighborhoods of $M$ such that $\bar{V} \subset$ int $U$. Let $\varepsilon>0$. By the construction of $V$, we have $\partial V \pi \varepsilon \subset$ int $V$ and $V \pi[0,2 \varepsilon] \subset \bar{V} \subset$ int $U$. Let $\pi_{i}$ be any net in $\Pi$ such that $\pi_{i} \rightarrow \pi$. We will first show that eventually $\partial V \pi_{i} \varepsilon \subset$ int $V$. Assume not. Then there is a subnet $\pi_{j}$ of $\pi_{i}$ and a net $x_{j}$ in $\partial V$ such that $x_{j} \pi_{j} \varepsilon \in X-$ int $V$. 
Since $\partial V$ is compact we may assume that the nets were chosen so that $x_{j} \rightarrow x \in \partial V$. Then we have $x_{j} \pi_{j} \varepsilon \rightarrow x \pi \varepsilon \in X$-int $V$ since $X$-int $V$ is closed. This is impossible because $x \pi \varepsilon \in \partial V \pi \varepsilon \subset$ int $V$. Hence, eventually $\partial V \pi_{i} \varepsilon \subset$ int $V$. In a similar manner we can show that eventually $\bar{V} \pi_{i}[0,2 \varepsilon] \subset$ int $U$. Hence, eventually, say for $i>i_{0}, \partial V \pi_{i} \varepsilon \subset$ int $V$ and $\bar{V}_{\pi_{i}}[0,2 \varepsilon] \subset$ int $U$. We now show that $V \pi_{i} R^{+} \subset U$ for $i>i_{0}$. Assume not. Then there is an $x \in \partial V$ and a $t>0$ such that $x \pi_{i} t \in \partial U$. Set $s=\sup \left\{\tau: x \pi_{i} \tau \in \partial V, 0 \leqq \tau<t\right\}$. Since $\partial V$ is compact, $x \pi_{i} s \in \partial V$. Moreover, $\left(x \pi_{i}(s, t)\right) \cap \bar{V}=\varnothing$. Since $x \pi_{i} s \in \partial V$, $\left(x \pi_{i} s\right) \pi_{i}(t-s)=x \pi t \in \partial U$, and $\bar{V} \pi_{i}[0,2 \varepsilon] \subset$ int $U$, we must have $t-s>2 \varepsilon$. But $\partial V \pi_{i} \varepsilon \subset$ int $U$. This contradicts

$$
\varnothing=\left(x \pi_{i}(s, t)\right) \cap V=\left(\left(x \pi_{i} s\right) \pi_{i}(0, t-s)\right) \cap V .
$$

This contradiction implies $V \pi_{i} R^{+} \subset U$ for $i>i_{0}$. It easily follows that $P(V) \subset \bar{U}=U$. The desired result now follows from Theorem 8 .

COROLlaRY 10. Let $M$ be a compact set which possesses a fundamental system of asymptotically stable neighborhoods. Then $M$ is totally stable.

Proof. The proof is an immediate consequence of Corollary 7 and Theorem 9.

Many questions arise which are, as of now, unanswered:

(1) Is $P$ transitive?

(2) If $M$ is totally stable, is $M$ absolutely stable?

(3) Is there a characterization of total stability similar to the characterization of stability under persistent perturbations in [5] and [6], e.g. does $M$ have a fundamental system of asymptotically stable neighborhoods?

REMARK. Instead of considering all nets in $\Pi$ which converge to $\pi$, we could have proven the same results with respect to a specific net $\pi_{i}$. This would yield a concept of "total stability with respect to $\pi_{i}$ ".

\section{BIBLIOGRAPHY}

1. J. Auslander and P. Seibert, Prolongations and stability in dynamical systems, Ann. Inst. Fourier (Grenoble) 14 (1964), fasc. 2, 237-267. MR 31 \#455.

2. O. Hájek, Dynamical systems in the plane, Academic Press, New York, 1968. MR 39 \#1767.

3. J. L. Kelley, General topology, Van Nostrand, Princeton, N.J., 1955. MR 16, 1136.

4. R. McCann, Another characteristization of absolute stability, Ann. Inst. Fourier (Grenoble) 21 (1971), fasc. 4, 175-177.

5. P. Seibert, A concept of stability in dynamical systems, Topological Dynamics, edited by J. Auslander and W. Gottschalk, Benjamin, New York, 1968.

6. - Stability under sustained perturbations and its generalization to continuous flow's, Acta Mexicana Ci. Tecn. 2 (1968), 154-165. (Spanish) MR 40 \#4560.

Department of Mathematics and Statistics, Case Western Reserve University, Cleveland, OHio 44106 